\title{
ON THE PROLONGATION OF VALUATIONS
}

\author{
BY \\ PETER ROQUETTE
}

\section{INTRODUCTION AND NOTATIONS}

1. Introduction. Let $k$ be a field and let $v$ be a valuation ring of $k$. We consider a finite algebraic extension field $K$ of $k$ and the valuation rings $V$ of $k$ lying above $v$ (i.e. $V \cap k=v$ ). Let $e_{V}$ and $f_{V}$ denote the ramification degree and the residue degree of $V$ over $k$. Then we shall prove the following fundamental inequality

$$
\sum_{V} e_{V} f_{V} \leqq n
$$

where $n$ denotes the degree of $K$ over $k$. This inequality is well known when $v$ and hence all the $V$ are of rank one. The main object of this paper is to show that the general case can be treated very similarly to the rank one case.

In II we give for valuations of arbitrary rank a theory of completions which does not seem to exist in the literature, although it is very easy and natural. This theory enables us to reduce the proof of (1) to the case where the ground field $k$ is complete (see the corollary to Lemma 5 in II, §8). However, for complete fields with respect to valuations of higher rank, Hensel's lemma is no longer true and therefore the usual proof of (1) for the complete case is no longer possible. For that reason we prove some kind of generalization of Hensel's lemma (corollary to Lemma 2 in II, §5) which will serve us as well, together with some simple facts on the composition of valuations (III).

One of the most important problems in valuation theory is to decide when equality holds in (1). If $v$ is discrete and of rank one, there are two criteria known: the first criterion requires that $K / k$ shall be separable. The second criterion requires that $k$ is either absolutely or relative to a subfield $\kappa$ of $v$ finitely generated and of dimension one $\left({ }^{1}\right)$. We are able to generalize these two results to discrete valuation rings of arbitrary finite rank $r$ in the following form:

If $v$ is of rank $r$ there is a chain

$$
v=v^{r} \subset v^{r-1} \subset \cdots \subset v^{1} \subset v^{0}=k
$$

of valuation rings $v^{i}$ of $k$ having rank $i$; they are uniquely determined by $v$

Received by the editors October 15, 1956.

(1) If $\kappa$ is a subfield of $k$ then the relative dimension is often called "degree of transcendency." The absolute dimension of $k$ is defined by means of the prime field $Q$ contained in $k$ as $\operatorname{dim}(k)=1+\operatorname{dim}(k / Q)$ if the characteristic of $k$ is zero, or as $\operatorname{dim}(k)=\operatorname{dim}(k / Q)$ if the characteristic of $k$ is a prime number. 
and may be called the components of $v$ (see e.g. Schilling, The theory of valuations, Chapter I, \$4). If $V$ lies above $v$ then $V$ is of the same rank $r$ as $v$. The $i$ th component $V^{i}$ of $V$ lies above $v^{i}$. Therefore the residue field $\Omega^{i}$ of $V^{i}$ is a finite algebraic extension field of the residue field $\mathfrak{f}^{i}$ of $v^{i}$. With these notations we can state the

FIRST CRITERION. If $v$ is discrete of rank $r$, and if for each valuation ring $V$ of $K$ lying above $v$ the field extensions $\Omega^{i} / \mathfrak{f}^{i}$ are separable for $i=0, \cdots, r-1$, then we have the degree formula

$$
\sum_{V} e_{V} f_{V}=n
$$

Thus the fact that (2) is not true in general is due to inseparability effects of the fields $\Re^{i} / \mathfrak{l}^{i}$ for $i=0, \cdots, r-1\left({ }^{2}\right)$.

Furthermore we shall prove the

SECOND CRITERION. Let $v$ be discrete of finite rank. Let $k$ be either absolutely or relative to a subfield $\kappa$ of $v$ finitely generated and assume that

$$
\operatorname{dim}(k)=\operatorname{dim}(\mathfrak{f})+\operatorname{rank}(v) .
$$

Then we have the degree formula (2).

Here, in the absolute case (where there is no constant field $\kappa$ ) the symbol $\operatorname{dim}(\cdots)$ in (3) means the absolute dimension. In the relative case it means the dimension relative to $\kappa$; note that $\kappa$ is mapped isomorphically into $\mathfrak{f}$ by the residue homomorphism of $v$ so that $\kappa$ may be regarded as a subfield of $\mathfrak{t}$.

In the course of the proof of the two criteria we will prove a theorem which axiomatically gives conditions under which (2) holds, (IV, \$12).

2. Notations. In order to avoid repetition we will consistently use without further explanations the following notations:

If a valuation ring $v$ of $k$ is given, then

$\mathfrak{p}$ is the maximal ideal of $v$;

$\mathfrak{t}=v / \mathfrak{p}$ is the residue field of $v$;

$\pi$ is the residue homomorphism (place) of $v$ onto $\mathfrak{l}$;

$k^{*}$ is the multiplicative group of $k$;

$g=k^{*} /(v-p)$ is the value group of $v$;

$\phi$ is the valuation of $v$, i.e. the natural homomorphism of $k^{*}$ onto $g$. We regard $g$ as a simply ordered group by defining $\phi(a) \leqq \phi(b)$ if $a b^{-1} \in v$.

As usual, we adjoin to $g$ an element 0 and put $\phi(0)=0$. We then have the triangle law

$$
\phi(a+b) \leqq \operatorname{Max}(\phi(a), \phi(b)) .
$$

If $K$ is another field, we will denote valuation rings of $K$ e.g. with $V$ and will

(2) It is not difficult to prove a relation of the form $\sum_{V} \delta_{V} e_{V} f_{V}=n$ which is true for every discrete valuation ring $v$, and where the weight $\delta_{V}$ is a divisor of the product of the degrees of inseparability of the field extensions $\Omega^{i} / \mathfrak{l}^{i}$ for $i=0, \cdots, r-1$. 
then also use capital letters for its prime ideal, residue homomorphism, valuation etc. If we consider several valuation rings of $K$ we will distinguish them by indices and will use the same indices for the prime ideals, residue homomorphisms, valuations, etc.

If $K$ is an extension field of a field $k$, and if $v=V \cap k$, then we define the ramification degree $e_{V}$ and the residue degree $f_{V}$ by

$$
e_{V}=[G: g], \quad f_{V}=[\Re: \ell] .
$$

Here, in the first formula, the quotient in brackets denotes the group theoretical index, and in the second formula it denotes the field theoretical degree. It is well known that both are finite. If we want to indicate what field extension we are referring to, then we write more precisely $e_{V}(K / k)$ and $f_{V}(K / k)$.

\section{Preliminaries on fields topologized by valuations}

3. Let $k$ be a field with a valuation ring $v$ and valuation $\phi$. The field $k$ may be topologized if we define as neighborhoods of zero the sets $v_{\epsilon}(\epsilon \in g)$ consisting of all $a \in k$ with $\phi(a) \leqq \epsilon$. If $a_{\epsilon}$ is in $k$ such that $\phi\left(a_{\epsilon}\right)=\epsilon$, then $v_{\epsilon}$ is precisely the principal ideal of $k$ with respect to $v$ determined by $a_{\epsilon}$. If we regard $k$ as a topological field we write $k_{v}$. As a topological space, $k_{v}$ is Hausdorff if and only if $v \neq k$.

Lemma 1. Let $v, v^{\prime}$ be valuation rings of $k$ and assume that not both of them are $=k$. Then $k_{v}=k_{v}$, if and only if there is a valuation ring $v^{\prime \prime} \neq k$ of $k$ which contains both $v$ and $v^{\prime}$.

Proof. (a) First we assume $v \subset v^{\prime}, v^{\prime} \neq k$ and show $k_{v}=k_{v^{\prime}}$. Since $v \subset v^{\prime}$ there exists an order-preserving homomorphism $s$ of $g$ onto $g^{\prime}$ such that

$$
s(\phi(a))=\phi^{\prime}(a) \text { for } a \in k^{*} .
$$

(See e.g. Schilling, The theory of valuations, pp. 16-17.) This shows that $v_{\epsilon} \subset v_{\epsilon^{\prime}}$ if $\epsilon^{\prime}=s(\epsilon)$. Hence the topology of $k_{v}$ is finer than the topology of $k_{v^{\prime}}$. To prove the converse, let $\epsilon \in g$ be given, and let $\epsilon^{\prime} \in g^{\prime}$ with $\epsilon^{\prime}<s(\boldsymbol{\epsilon})$. Such an element $\epsilon^{\prime}$ exists, since by assumption $v^{\prime} \neq k$ and hence $g^{\prime} \neq 1$. With this $\epsilon^{\prime}$ we have $v_{\epsilon^{\prime}} \subset v_{\epsilon}$. For, if $a \notin v_{\epsilon}$, i.e. if $\phi(a)>\epsilon$, it follows $\phi^{\prime}(a)=s(\phi(a))$ $\geqq s(\boldsymbol{\epsilon})>\epsilon^{\prime}$, i.e. $a \notin v_{\epsilon^{\prime}}$.

(b) From (a) it follows: If $v, v^{\prime}$ are as in the lemma and if there exists a valuation ring $v^{\prime \prime} \neq k$ containing $v$ and $v^{\prime}$ then $k_{v}=k_{v^{\prime}}$.

(c) Now we assume that $k_{v}=k_{v^{\prime}}$ and construct a valuation ring $v^{\prime \prime}$ with the required properties. Let, say, $v \neq k$, so that $g \neq 1$ and hence there exist elements $\epsilon \in g$ with $\epsilon<1$.

The valuation ring $v^{\prime}$ itself is a neighborhood of zero in $k_{v^{\prime}}$, belonging to the identity element of $g^{\prime}$. Hence, since $k_{v}=k_{v^{\prime}}$ there exist arbitrary small elements $\epsilon<1$ in $g$ such that $v_{\epsilon} \subset v^{\prime}$. Let $p^{\prime \prime}$ be the union of those ideals $v_{\epsilon}$. It is easy to see that $p^{\prime \prime}$ is a prime ideal of $v$. The quotient ring $v^{\prime \prime}$ of $v$ with respect to $\mathfrak{p}^{\prime \prime}$ is therefore a valuation ring of $k$ and $\mathfrak{p}^{\prime \prime}$ is its prime ideal. Since $\mathfrak{p}^{\prime \prime}$ is 
contained in both $v$ and $v^{\prime}$ by construction, $v^{\prime \prime}$ contains both $v$ and $v^{\prime}$. Since $\mathfrak{p}^{\prime \prime} \neq 0$ by construction, we have $v^{\prime \prime} \neq k$. Q.E.D.

COROLlary. Let $S$ be a finite set of valuation rings of $k$ and assume that there is $a v$ in $S$ with $v \neq k$. Then there exists a valuation ring $v^{\prime \prime} \neq k$ of $k$ containing all the $v \in S$ if and only if $k_{v}=k_{v^{\prime}}$ for each pair $v, v^{\prime}$ of $S$. If this is so, among the $v^{\prime \prime}$ in question there exists a uniquely determined smallest valuation ring $v(S)$ which we shall call the conductor of $S$.

The first assertion follows from Lemma 1 by induction. The second assertion follows from the well known fact that the valuation rings of $k$ containing a given valuation ring $v$ of $k$ are simply ordered by inclusion; therefore the intersection of all the $v^{\prime \prime}$ considered in the corollary is again a $v^{\prime \prime}$.

4. Completions. Let $k$ be a field with a valuation ring $v$. Let us assume $v \neq k$ so that $k_{v}$ is a Hausdorff space. Then, by the general theory of topological algebra, there is a uniquely determined completion $\vec{k}_{v}$ of $k_{v}$. This is a topological field containing $k_{v}$ (more precisely: containing an isomorphic image of $k_{v}$ ) as a dense subfield. The topology of $\bar{k}_{v}$ may be defined by a valuation ring $\bar{v}$ of $\vec{k}_{v}$ lying above $v$, and $\bar{v}$ is the completion of $v$ in $\bar{k}_{v}$. The value groups of $v$ and $\bar{v}$ coincide, as well as the residue fields of $v$ and $\bar{v}$. That is, we have

$$
e_{\bar{v}}\left(\bar{k}_{v} / k\right)=1, \quad f_{\bar{v}}\left(\bar{k}_{v} / k\right)=1 .
$$

The proofs of all these facts may easily be obtained by the classical methods (see e.g. Bourbaki, Topologie generale, fascicule de resultats, §10, 16-20).

If $k_{v}=k_{v}$, then $\bar{k}_{v}=\bar{k}_{v^{\prime}}$ (more precisely: if the identity isomorphism of $k$ defines a topological isomorphism of $k_{v}$ onto $k_{v}$, then it can be extended uniquely to topological isomorphism of $\bar{k}_{v}$ onto $\bar{k}_{v^{\prime}}$ leaving $k$ fixed). Conversely, if $\vec{k}_{v}$ and $\bar{k}_{v^{\prime}}$ are topologically isomorphic over $k$, then $k_{v}=k_{v^{\prime}}$.

Now let $K$ be a finite algebraic extension field of $k$ and $V$ a valuation ring of $K$ lying above $v$. Then we claim first that

$$
k_{v} \subset K_{V},
$$

i.e. the topology of $k_{v}$ is induced by the topology of $K_{V}$. To prove this, we regard as usual $g$ as a subgroup of $G$ and $\Phi$ as a prolongation of $\phi$. Then for each $\epsilon \in g$ we have $v_{\epsilon}=V_{\epsilon} \cap k$ by definition. Hence it suffices to show that to each element $E \in G$ there exists an $\epsilon \in g$ with $V_{\epsilon} \subset V_{E}$, i.e. $\epsilon \leqq E$. Without restriction we may assume $E \leqq 1$; then $E^{m} \leqq E$ for each positive integer $m$. Taking $m$ to be the exponent of $G$ modulo $g$ we have $G^{m} \subset g$, hence $E^{m}=\epsilon$ is as required. This proves (5).

From (5) we conclude that $\bar{k}_{v} \subset \bar{K}_{V}$ (more precisely: there is an isomorphism of $\bar{k}_{v}$ into $\left.\bar{K}_{V}\right)$. The completion $\bar{V}$ of $V$ is a valuation ring lying above $\bar{v}$. Since the ramification degree and the residue degree behave multiplicatively under composition of field extensions we conclude from (4) and the corresponding formulas for $\bar{V}$ that 


$$
e_{V}(K / k)=e_{\bar{V}}\left(\bar{K}_{V} / \bar{k}_{v}\right), \quad f_{V}(K / k)=f_{\bar{V}}\left(\bar{K}_{V} / \bar{k}_{v}\right) .
$$

Thus in computing the ramification degree and the residue degree one may always lift $v$ and $V$ to their completions.

5 . We use the same assumptions and notations as in $\$ 4$. We will always identify $\bar{k}_{v}$ with its isomorphic image in $\bar{K}_{V}$. On the other hand it will be convenient not to identify $K_{V}$ with its isomorphic image in $\bar{K}_{V}$; so let us denote by $\sigma_{V}$ the canonical injection of $K_{V}$ into $\bar{K}_{V}$.

We first assert that

$$
\bar{K}_{V}=\sigma_{V}\left(K_{V}\right) \cdot \bar{k}_{v},
$$

where the right hand side denotes the compositum of the two fields. This amounts to show that $\sigma_{V}\left(K_{V}\right) \cdot \bar{k}_{v}$ is complete under the topology induced by $\bar{V}$. Since $\sigma_{V}\left(K_{V}\right) \cdot \bar{k}_{v}$ is a finite algebraic extension of $\bar{k}_{v}$ this follows from

Lemma 2. Let $k$ be a field with a valuation ring $v \neq k$ and assume that $k_{v}$ is complete. If $K$ is a finite algebraic extension field of $k$ and $V$ a valuation ring of $K$ lying above $v$ then $K_{V}$ is complete too.

In this proof we will not use the fact that $K$ is a field but only that $K$ is a finite dimensional vector space over $k$. Accordingly, we will only use the fact that the valuation $\Phi$ of $K$ is a function on $K$ with values in some ordered group $G$ containing $g$ and of the same ordering type as $g$ such that

(a) $\Phi(a) \neq 0$ if $a \neq 0$;

(b) $\Phi(a+b) \leqq \operatorname{Max}(\Phi(a), \Phi(b))$;

(c) $\Phi(c a)=\phi(c) \Phi(a)$ if $c \in k$.

Let us call a vector space $K$ over $k$ in which such a function is defined a normed vector space. Then we may rephrase Lemma 2 as follows:

Let $k$ be a field with a valuation ring $v \neq k$ and valuation $\phi$. Let $K$ be a finite dimensional vector space over $k$ with a norm $\Phi$. Then, if $k_{v}$ is complete, $K$ is complete too with respect to the topology induced by $\Phi$.

Proof. Let $u_{1}, \cdots, u_{n}$ be a basis of $K / k$. For every element $a=\sum_{i} a_{i} u_{i}$ of $K$ we put

$$
\Phi^{\prime}(a)=\underset{i}{\operatorname{Max}} \phi\left(a_{i}\right) .
$$

It is easily seen that $\Phi^{\prime}(a)$ is a norm of $K$. Furthermore, if we identify $a$ with the element $\left(a_{1}, \cdots, a_{n}\right)$ of the product space $k_{v}^{n}$ and hence $K$ with $k_{v}^{n}$ then the topology defined by $\Phi^{\prime}$ is precisely the product topology. Since $k_{v}$ is complete it follows that $K$ is complete with respect to $\Phi^{\prime}$.

Hence we have to show that the topologies of $K$ determined by $\Phi$ and $\Phi^{\prime}$ coincide. That means there are elements $N$ and $N^{\prime}$ in $G$ such that for each $a \in K$ we have

$$
N \cdot \Phi^{\prime}(a) \leqq \Phi(a) \leqq N^{\prime} \cdot \Phi^{\prime}(a) .
$$


The second inequality is an immediate consequence of the axioms (b)-(c) for $\Phi$ and the definition of $\Phi^{\prime}$. So we have only to deal with the first inequality of (8).

The case $n=1$ being trivial we may assume $n>1$ and the theorem already proved for $n-1$. In order to prove it for $n$, it suffices to prove for each $i=1, \cdots, n$ a relation of the form

$$
N_{i} \cdot \phi\left(a_{i}\right) \leqq \Phi(a)
$$

since we then can take $N=\operatorname{Min}_{i} N_{i}$. In proving this relation the index $i$ remains fixed so let us write $i=1$.

For each $a \in K$ put $a=a_{1} u_{1}+b$ where $b$ belongs to the subspace $L$ of dimension $n-1$ spanned by $u_{2}, \cdots, u_{n}$. Then we have to show the existence of an element $N_{1} \in G$ such that

$$
N_{1} \leqq \Phi\left(a_{1}^{-1} a\right)=\Phi\left(u_{1}-b^{\prime}\right)
$$

where we put $b^{\prime}=-a_{1}^{-1} b \in L$. This means that there is a neighborhood of $u_{1}$ of a certain radius $N_{1}$ which does not meet $L$. In other words: we have to show that $L$ is closed under the topology determined by $\Phi$.

Now, $L$ has dimension $n-1$. Therefore we may apply induction hypothesis and conclude that $L$ is complete with respect to $\Phi$. As a complete subspace of $K$ it is closed. Q.E.D. $\left(^{3}\right)$.

Thus we have proved Lemma 2 and hence (7). From this proof, in particular from (8), we conclude that the topology of $K_{V}$ does not depend on $V$ at all but is already determined by $v$. Hence we get the

Corollary. Under the assumptions of Lemma 2 we have $K_{V}=K_{V^{\prime}}$ for any two valuation rings $V, V^{\prime}$ of $K$ lying above $v$.

REMARK. If $v$ is of rank one then each $V$ is also of rank one and hence a maximal subring of $K$. Therefore in this case the above corollary together with Lemma 1 shows that there is only one valuation ring $V$ of $K$ lying above $v$ provided $k_{v}$ is complete. In classical valuation theory this is a consequence of Hensel's lemma, and conversely. Therefore the above corollary may be viewed as a generalization of Hensel's lemma to valuation rings of arbitrary rank.

6. Characterization of nonequivalent prolongations by isomorphisms. Again let $k$ be a field with a valuation ring $v \neq k$. Let $K$ be a finite algebraic extension field of $k$. Let us call two valuation rings $V, V^{\prime}$ of $K$ equivalent if they define the same topology of $K$, i.e. if $K_{V}=K_{V^{\prime}}$. On the other hand, let us call two isomorphisms $\sigma, \sigma^{\prime}$ of $K / k$ into extension fields of $\vec{k}_{v}$ equivalent if there exists a $\bar{k}_{v}$-isomorphism $\mu$ of the compositum $\sigma(K) \cdot \bar{k}_{v}$ onto $\sigma^{\prime}(K) \cdot \bar{k}_{v}$ such that $\mu(\sigma(a))=\sigma^{\prime}(a)$ for $a \in K$.

( $\left.{ }^{3}\right)$ This proof was given by Artin in his lecture for the special case where $g$ is a subgroup of the multiplicative group of positive real numbers. 
I.EMM. 3. If $V$ ranges over a full system of nonequivalent valuation rings of $K$ lying above $v$ then $\sigma_{V}$ ranges over a full system of nonequivalent isomorphisms of $K / k$ into extension fields of $\bar{k}_{v}$.

I.EMMA 4. If $V$ ranges over the set of valuation rings of $K$ lying above $v$ and equivalent to a fixed valuation ring $V_{0}$ then their completions $\bar{V}$ in $\bar{K}_{V_{0}}$ range over the set of all valuation rings lying above $\bar{v}$.

Proof of Lemma 3. (a) Assume that $\sigma_{V}$ and $\sigma_{V}$, are equivalent, and let $\mu$ be the isomorphism as required in the definition of equivalence. By (7) it follows that $\mu$ is an isomorphism (in the algebraic sense) of the field $\bar{K}_{V}$ onto $\bar{K}_{V^{\prime}}$. From the corollary to Lemma 2 it follows that $\mu$ is even a topological isomorphism. Hence $V$ and $V^{\prime}$ are equivalent.

(b) Assume that $V$ and $V^{\prime}$ are equivalent. Then we may identify $\bar{K}_{V}$ and $\bar{K}_{V}$, so that we have $\sigma_{V}=\sigma_{V}$.

(c) It remains to show that to each isomorphism $\sigma$ of $K$ into an extension ficld of $\bar{k}_{v}$ there belongs a valuation ring $V$ of $K$ lying above $v$ such that $\sigma_{V}$ and $\sigma$ are equivalent. Put $K^{\prime \prime}=\sigma(K) \cdot \bar{k}_{v}$ and let $V^{\prime \prime}$ be a valuation ring of $K^{\prime \prime}$ lying above $\bar{v}$. Consider the contraction $V^{\prime \prime} \cap \sigma(K)$ and let $V$ be the valuation ring of $K$ mapping onto $V^{\prime \prime} \cap \sigma(K)$ under $\sigma$. Then $V$ lies above $v$. By construction, $\sigma$ is an isomorphism of $K_{V}$ into $K_{V^{\prime \prime}}^{\prime \prime}$. Thus, since $K_{V^{\prime \prime}}^{\prime \prime}$ is complete by Lemma 2, $\sigma$ can be extended by continuity to an isomorphism $\mu$ of $\bar{K}_{V}$ into $K_{V^{\prime \prime}}^{\prime \prime}$ leaving $\vec{k}_{v}$ elementwise fixed. We have $\mu\left(\sigma_{V}(a)\right)=\sigma(a)$ by construction of $\mu$; hence $\sigma_{V}$ and $\sigma$ are indeed equivalent. Q.E.D.

Proof of Lemma 4. If $V$ is equivalent to $V_{0}$ then we can identify $\bar{K}_{V}=\bar{K}_{V_{0}}$. Then $\bar{V}$, the completion of $V$ in $\bar{K}_{V_{0}}$, is a valuation ring of $\bar{K}_{V_{0}}$ defining the topology of $\bar{K}_{V_{0}}$ and lying above $\bar{v}$. Since $\bar{V}$ contracts to $V$ in $K$ the relation $V \leftrightarrow \bar{V}$ is one-one. Hence it remains to show that each valuation ring $V^{\prime \prime}$ of $\bar{K}_{V_{0}}$ lying above $\bar{v}$ is the completion of some $V$ lying above $v$.

Consider the contraction $V^{\prime \prime} \cap \sigma_{V_{0}}(K)$ and let $V$ be the valuation ring which maps onto it under $\sigma_{V_{0}}$. As shown in (c) of the above proof, we may identify $\bar{K}_{V}$ and $\bar{K}_{V_{0}}$ (by means of a uniquely determined isomorphism $\mu$ ). Then, by construction, $\bar{V}$ will be contained in $V^{\prime \prime}$. On the other hand, $\bar{V}$ and $V^{\prime \prime}$ both are lying above $\bar{v}$. Therefore our contention $\bar{V}=V^{\prime \prime}$ follows from the following general principle:

7. Proposition 1. Let $k$ be a field with a valuation ring $v$ and $K$ an extension field of $k$. Let $V$ and $V^{\prime}$ be two valuation rings of $K$ lying above $v$ and assume that $[G: g]$ is finite. Then, if $V \subset V^{\prime}$, we have $V=V^{\prime}$.

Proof. If $V \subset V^{\prime}$ there is an order preserving homomorphism $s$ of $G$ onto $G^{\prime}$ such that $s(\Phi(a))=\Phi^{\prime}(a)$ for $a \in K^{*}$. Since $\Phi$ and $\Phi^{\prime}$ are both prolongations of $\phi$ it follows that $s$ leaves $g$ elementwise fixed. Since $[G: g]$ is finite it follows $b^{[G: \theta]}=1$ for each element $b \in G$ which is in the kernel of $s$. Since $G$ as a simply ordered group does not contain elements $\neq 1$ of finite order it follows $b=1$. Hence $s$ is an isomorphism, i.e. $V=V^{\prime}$. Q.E.D. 
8. The local degrees and the defects of inseparability. Now, in the same situation as in $\S 6$, let us define for each $V$ lying above $v$ the local degree

$$
n_{V}=\left[\bar{K}_{V}: \bar{k}_{v}\right]
$$

and the inseparability defect

$$
d_{V}=[K: k]_{i} /\left[\bar{K}_{V}: \bar{k}_{v}\right]_{i}
$$

where the index $i$ means "degree of inseparability." From (7) it follows that $d_{V}$ is an integer. Both $d_{V}$ and $n_{V}$ do not depend on the choice of $V$ among its equivalence class.

Lemma 5. If $n=[K: k]$ then we have the formula

$$
\sum_{V}^{\prime} d_{V} n_{V}=n
$$

where the prime means that $V$ ranges over a full system of nonequivalent valuation rings of $K$ lying above $v$.

Proof. For simplicity, let us write $k^{\prime}=\bar{k}_{v}$ and $\sigma=\sigma_{V}$. Then, using (7) and the Lemma 3, we see that the formula to be proved may be written as

$$
\sum_{\sigma}^{\prime} d_{\sigma} n_{\sigma}=n
$$

where

$$
\begin{aligned}
& d_{\sigma}=[K: k]_{i} /\left[\sigma(K) \cdot k^{\prime}: k^{\prime}\right]_{i}, \\
& n_{\sigma}=\left[\sigma(K) \cdot k^{\prime}: k^{\prime}\right]
\end{aligned}
$$

and where $\sigma$ ranges over a full system of nonequivalent isomorphisms of $K / k$ over $k^{\prime}$.

Formula $(\mathrm{F})$ now has nothing to do with valuations and is a purely algebraic statement. It is well known and proved e.g. as follows:

By the definition of $d_{\sigma}$ and $n_{\sigma}$, (F) may be rewritten as

$$
\sum_{\sigma}^{\prime}\left[\sigma(K) \cdot k^{\prime}: k^{\prime}\right]_{s}=[K: k]_{s}
$$

where the index $s$ means "degree of separability." Now, if $K_{0}$ is the separable closure of $k$ in $K$ then $[K: k]_{s}=\left[K_{0}: k\right]$. On the other hand, $\sigma\left(K_{0}\right) \cdot k^{\prime}$ is the separable closure of $k^{\prime}$ in $\sigma(K) \cdot k^{\prime}$ and therefore $\left[\sigma(K) \cdot k^{\prime}: k^{\prime}\right]_{s}=\left[\sigma\left(K_{0}\right) \cdot k^{\prime}: k^{\prime}\right]$. Therefore, we may assume $K=K_{0}$, i.e. $K / k$ separable, and have to prove the formula

$$
\sum_{\sigma}^{\prime} n_{\sigma}=n
$$

in this case.

Let $a$ be a generating element of $K / k$; then $\sigma(a)$ generates $\sigma(K) \cdot k^{\prime} / k^{\prime}$ for each $\sigma$. If $\sigma$ ranges over a full system of nonequivalent isomorphisms of $K / k$ 
over $k^{\prime}$ then $\sigma(a)$ ranges over a full system of nonconjugate elements over $k^{\prime}$. Hence the irreducible polynomials $f_{\sigma}(x)$ of $\sigma(a)$ over $k^{\prime}$ range over the distinct $k^{\prime}$-irreducible factors of $f(x)$, the irreducible polynomial of $a$ over $k$. Because $a$ is separable, we have therefore $f(x)=\prod_{\sigma}^{\prime} f_{\sigma}(x)$. Hence we get the desired formula by comparing degrees on both sides of this polynomial equation.

CoROllary. Let $K / k$ be a finite algebraic extension of degree $n$, and let $v \neq k$ be a valuation ring of $k$. Then, in order to prove the fundamental inequality (1) for $K / k$ and $v$ it is sufficient to prove it for each $\bar{K}_{V} / \bar{k}_{v}$ and $\bar{v}$, where $V$ ranges over the valuation rings of $K$ lying above $v$.

Of course, it suffices to let $V$ range over a full system of nonequivalent valuation rings lying above $v$.

Proof. By Lemma 5 we have $\sum_{V}^{\prime} n_{V} \leqq n$. This shows that in order to prove (1) it is sufficient to prove for each fixed $V_{0}$ the inequality

$$
\sum_{V \sim V_{0}} e_{V} f_{V} \leqq n_{V_{0}}
$$

where the sum is to be extended over all valuation rings $V$ of $K$ lying above $v$ and equivalent to $V_{0}$. Using Lemma 4 and (6) this amounts to showing that

$$
\sum_{\bar{V}} e_{\bar{V}} f_{\bar{V}} \leqq n_{V_{0}}
$$

where now $\bar{V}$ ranges over all the valuation rings of $\bar{K}_{V_{0}}$ lying above $\bar{v}$. Q.E.D.

\section{ON THE COMPOSITION OF VALUATIONS}

9. We consider the following situation. Let be:

$K / k$ a finite algebraic field extension;

$V^{\prime}$ a valuation ring of $K$ with residue homomorphism $\Pi^{\prime}$;

$\mathfrak{\Omega}^{\prime} / \mathfrak{f}^{\prime}$ the finite algebraic field extension obtained from $K / k$ by applying $\Pi^{\prime}$, i.e. $\Omega^{\prime}=\Pi^{\prime}\left(V^{\prime}\right), \mathfrak{f}^{\prime}=\Pi^{\prime}\left(V^{\prime} \cap k\right)$;

$v$ a given valuation ring of $k$ with $v \subset V^{\prime}$;

$v_{1}$ the valuation ring of $\mathfrak{t}^{\prime}$ which is the image of $v$ under the homomorphism $\Pi^{\prime}$.

Lemma 6. The valuation rings $V$ of $K$ contained in $V^{\prime}$ correspond one-one to the valuation rings $V_{1}$ of $\Omega^{\prime}$ by the relation $V_{1}=\Pi^{\prime}(V)$. In this correspondence, inclusion relations are preserved in both directions. $V$ lies above $v$ if and only if $V_{1}$ lies above $v_{1}$. If this is so, we have the formulae

$$
f_{V}(K / k)=f_{V_{1}}\left(\Omega^{\prime} / \mathfrak{t}^{\prime}\right), \quad e_{V}(K / k)=e_{V^{\prime}}(K / k) \cdot e_{V_{1}}\left(\Omega^{\prime} / \mathfrak{t}^{\prime}\right) .
$$

Proof. (a) If $V \subset V^{\prime}$ then $\mathfrak{B}^{\prime} \subset \mathfrak{B}$, and conversely. Hence the valuation rings of $K$ contained in $V^{\prime}$ are exactly the valuation rings contained in $V^{\prime}$ and containing the kernel $\mathfrak{B}^{\prime}$ of $\Pi^{\prime}$. Therefore, the relation $V \leftrightarrow V_{1}$ with 


$$
V_{1}=\Pi^{\prime}(V), \quad V=\bar{\Pi}^{-1}\left(V_{1}\right)
$$

is indeed one-one and preserves inclusion relations in both directions.

(b) Let $\pi^{\prime}$ be the residue homomorphism induced by $\Pi^{\prime}$ in $k$. Then, by the same argument as in $(a)$, we have

$$
V_{1} \cap \mathfrak{f}^{\prime}=\pi^{\prime}(V \cap k), \quad V \cap k=\boldsymbol{\pi}^{-1}\left(V_{1} \cap \mathfrak{f}^{\prime}\right),
$$

if $V$ and $V_{1}$ correspond under $\Pi^{\prime}$. Hence we have indeed $V \cap k=v$ if and only if $V_{1} \cap \mathfrak{f}^{\prime}=v_{1}$.

(c) Let $\sigma$ be the isomorphism of $\Omega$ onto $\Omega_{1}$ defined

$$
\sigma(\Pi(a))=\Pi_{1}\left(\Pi^{\prime}(a)\right) \quad \text { for } a \in V .
$$

If $V$ lies above $v$ then $V_{1}$ lies above $v_{1}$ and therefore $\Pi, \Pi_{1}, \Pi^{\prime}$ are prolongations of $\pi, \pi_{1}, \pi^{\prime}$ respectively. Hence

$$
\sigma(\pi(a))=\pi_{1}\left(\pi^{\prime}(a)\right) \quad \text { for } a \in v .
$$

Consequently, we have $\sigma(\mathfrak{t})=\mathfrak{f}_{1}$. Hence the field extension $\Re / \mathfrak{l}$ is mapped under $\sigma$ isomorphically onto $\Omega_{1} / \mathfrak{t}_{1}$ which proves $f_{V}=f_{V_{1}}$.

(d) Consider the value groups $G, G^{\prime}, G_{1}$ and the exact sequence

$$
1 \rightarrow G_{1} \rightarrow G \rightarrow G^{\prime} \rightarrow 1
$$

where the injection of $G_{1}$ into $G$ is defined by

$$
\Phi_{1}\left(\Pi^{\prime}(a)\right) \rightarrow \Phi(a) \quad \text { for } a \in V^{\prime}-\mathfrak{P}^{\prime}
$$

and the surjection of $G$ onto $G^{\prime}$ by

$$
\Phi(a) \rightarrow \Phi^{\prime}(a) \quad \text { for } a \in K^{*} .
$$

There is a similar exact sequence for $g, g_{1}, g^{\prime}$. Now, if $V$ lies above $v$, then $V_{1}$ lies above $v_{1}$, and therefore $\Phi, \Phi_{1}, \Phi^{\prime}$ are prolongations of $\phi, \phi_{1}, \phi^{\prime}$ respectively. Therefore the following diagram is commutative and exact:

$$
\begin{gathered}
1 \rightarrow G_{1} \rightarrow G \rightarrow G^{\prime} \rightarrow 1 \\
\uparrow \uparrow \uparrow \\
1 \rightarrow g_{1} \rightarrow g \rightarrow g^{\prime} \rightarrow 1
\end{gathered}
$$

where the vertical arrows mean the inclusion relations. From this we deduce by an elementary group theoretical theorem, known as "Artin's lemma," the relation $[G: g]=\left[G^{\prime}: g^{\prime}\right]\left[G_{1}: g_{1}\right]$. This is exactly the second assertion of (9). Q.E.D.

\section{Proof of the Results Stated in the introduction}

10. The fundamental inequality. We consider the situation described in the introduction, namely:

$K / k$ a finite algebraic field extension of degree $n$, 
$v$ a valuation ring of $k$,

$V$ ranges over the valuation rings of $K$ lying above $v$.

We are going to prove the fundamental inequality (1). We may exclude the trivial case $v=k$, thus assuming $v \neq k$ so that the results of II are applicable. If $n=1$ there is nothing to prove. So we may assume $n>1$ and the inequality already proved for smaller values of $n$.

If not all the $V$ lying above $v$ are equivalent then by Lemma 5 we have $n_{V}<n$ for each $V$. The corollary to Lemma 5 together with the induction hypothesis then show that (1) is true. It is our purpose now to reduce the general case, where all the $V$ may be equivalent, to the case where they are not.

To that end, consider the smallest valuation ring $V^{\prime}$ of $K$ containing all the $V$ lying above $v$. Then we have the situation of Lemma 6: let us use the notations introduced there. By the minimal choice of $V^{\prime}$ we learn from Lemma 6 that there is no valuation ring $\neq \Omega^{\prime}$ of $\Omega^{\prime}$ containing all the $V_{1}$ lying above $v_{1}$. Therefore, the corollary of Lemma 1 shows that there are only two cases possible $\left({ }^{4}\right)$ :

Either there is some $V_{1} \neq \Omega^{\prime}$; then not all the $V_{1}$ lying above $v_{1}$ are equivalent. Since $\left[\Omega^{\prime}: \mathfrak{f}^{\prime}\right] \leqq n$, we have for $v_{1}$ the case we dealt with above; so we conclude by the induction hypothesis

$$
\sum_{V_{1}} e_{V_{1}}\left(\Re^{\prime} / \mathfrak{f}^{\prime}\right) f_{V_{1}}\left(\Re^{\prime} / \mathfrak{t}^{\prime}\right) \leqq\left[\Re^{\prime}: \mathfrak{f}^{\prime}\right]
$$

where $V_{1}$ ranges over the valuation rings of $\Omega^{\prime}$ lying above $v_{1}$.

Or else there is only one $V_{1}$ lying above $v_{1}$, and we have $V_{1}=\Omega^{\prime}$. In this case the formula (10) is trivially true.

So in any case we have shown that (10) holds. We now note that $f_{V^{\prime}}(K / k)$ $=\left[\Omega^{\prime}: \mathfrak{f}^{\prime}\right]$. Therefore, multiplying $(10)$ by $e_{V^{\prime}}(K / k)$ and using Lemma 6 we see that in order to prove (1) it is sufficient to prove

$$
e_{V^{\prime}}(K / k) f_{V^{\prime}}(K / k) \leqq n \text {. }
$$

This formula, in which only one valuation ring $V^{\prime}$ occurs, is easy to prove and well known from general valuation theory so that we will not repeat its proof here. (See e.g. Schilling, The theory of valuations, p. 21, Lemma 18.) Q.E.D.

11. For later purposes we will now prove the following property of the valuation ring $V^{\prime}$ constructed in this proof. We will show that $V^{\prime}$ is the only

(4) REMARK. In applying the corollary of Lemma 1 we use the fact that given a valuation ring $v$ and a finite algebraic extension of its quotient field, there are only a finite number of valuation rings in it lying above $v$. This is not difficult to prove. However, it is not really necessary here. On the contrary, it is a consequence of the inequality (1) if one states that inequality in the following form: for any finite number of valuation rings $V$ lying above $v$ (1) holds. In proving this, one has to start with any finite number of valuation rings lying above $v$ and then carry through the same proof as given in the text. Then one arrives at a finite number of $V_{1}$ so that the corollary of Lemma 1 is applicable. 
valuation ring of $K$ lying above $V^{\prime} \cap k$. By construction, $V^{\prime}$ contains all valuation rings $V$ of $K$ lying above $v$. Therefore our contention follows from the

Lemma 7. Let $K / k$ be a finite algebraic extension and let $v$ be a valuation ring of $k$. Let $V^{\prime}$ be a valuation ring of $K$ containing all $V$ lying above $v$. Then $V^{\prime}$ is the only valuation ring of $K$ lying above $V^{\prime} \cap k$.

Proof. Let $V^{\prime \prime}$ lie above $V^{\prime} \cap k=v^{\prime}$. Then $\Omega^{\prime \prime} / \mathfrak{f}^{\prime}$ is a finite algebraic extension. Let us choose a valuation ring $W$ of $\Omega^{\prime \prime}$ lying above $v_{1}$. By Lemma 6 , applied to $V^{\prime \prime}$, there corresponds to $W$ a valuation ring $V$ of $K$ lying above $v$ and contained in $V^{\prime \prime}$. On the other hand, we have $V \subset V^{\prime}$ by our hypothesis. So $V$ is contained in both $V^{\prime}$ and $V^{\prime \prime}$. Therefore we have either $V^{\prime} \subset V^{\prime \prime}$ or $V^{\prime \prime} \subset V^{\prime}$. Since both $V^{\prime}, V^{\prime \prime}$ lie above $v^{\prime}$ we have $V^{\prime}=V^{\prime \prime}$ by our Proposition 1 in $\$ 7$. Q.E.D.

12. Now we investigate the proof given in $\S 10$ in order to see in which cases we can deduce equality in (1). We consider a class $\delta$ of pairs $(v, K)$ consisting of a valuation ring $v$ and a finite algebraic extension field $K$ of the quotient field $k$ of $v$. We want to write down conditions for $\delta$ which imply that for each $(v, K)$ in $\delta$ the inequality (1) is actually an equality.

First we see because of Lemma 5 that the following condition is necessary:

(i) If $(v, K)$ is in $\&$ then $d_{V}(K / k)=1$ for each valuation ring $V$ of $K$ lying above $v$.

Furthermore, in view of the corollary to Lemma 5 we require:

(ii) If $(v, K)$ is in $\delta$ then the completion $\left(\bar{v}, \bar{K}_{V}\right)$ is in $\delta$ too for each valuation ring $V$ of $K$ lying above $v$.

If these conditions are satisfied then a glance at its proof shows that the corollary to Lemma 5 may be translated to our situation as follows:

In order to prove the equality in (1) for a pair $(v, K)$ in $S$ it is sufficient to prove it for each $\left(\bar{v}, \bar{\Omega}_{V}\right)$ in $\mathrm{s}$.

Now, in the proof given in $\$ 10$ let us try to use this statement instead of the corollary to Lemma 5 . In order to carry out all the induction arguments in that proof we see that we have to require further:

(iii) If $(v, K)$ is in $S$ and if $V^{\prime}$ is a valuation ring of $K$ containing $v$, then, putting $v^{\prime}=V^{\prime} \cap k$ and $v_{1}=\pi^{\prime}(v)$, the pairs $\left(v^{\prime}, K\right)$ and $\left(v_{1}, \Omega^{\prime}\right)$ are in $\mathrm{S}$.

Let (i)-(iii) be satisfied. Then we see that in order to prove equality in (1) for all pairs in $\delta$ we can make the same induction proof as in $\S 10$ replacing $\leqq$ by $=$ whenever the former shows up as a consequence of the induction hypothesis. There is but one place in the proof where $\leqq$ shows up for another reason, namely the formula (11). Therefore, in view of what we have shown in $\S 11$ about $V^{\prime}$, we have still to require the following property of $\delta$ :

(iv) If $(v, K)$ is in $S$ and if there is only one valuation ring $V$ of $K$ lying above $v$ then $e_{V} f_{V}=n$.

Hence we have the following 
THEOREM 1. Let $S$ be a class of pairs $(v, K)$ where $v$ is a valuation ring and $K$ is a finite algebraic extension field of the quotient field $k$ of $v$. If $\&$ satisfies the Axioms (i)-(iv) then we have for each $(v, K)$ in $\mathrm{S}$ the degree formula (2).

13. Proof of the two criteria. Consider the class $D$ of pairs $(v, K)$ such that $v$ is discrete. Then it is trivial that $D$ satisfies the conditions (ii) and (iii).

First we prove:

Lemma 8. If a subclass $S$ of $D$ satisfies (i)-(iii) then it satisfies (iv).

Proof. Let $(v, K)$ in $\mathcal{S}$ and assume there is only one valuation ring $V$ of $K$ lying above $v$. If rank $(v)=0$ then $v=k$ and there is nothing to prove. If rank $(v)=1$ it follows from Lemma 5 and (i), (ii) that we may assume that $k$ is complete with respect to $v$. For extensions of complete fields with respect to a discrete rank one valuation ring the equality $e_{V} f_{V}=n$ is well known (see e.g. Schilling, loc. cit., p. 55, (i)).

If rank $(v)>1$ we use induction on the rank. Let $v \subset v^{\prime}$ such that rank $(v)$ $>\operatorname{rank}\left(v^{\prime}\right)>0$. Then there is only one $V^{\prime}$ above $v^{\prime}$ (see Lemma 7). Therefore, by (iii) and the induction hypothesis, we have $e_{V} f_{V^{\prime}}=n$. On the other hand, we consider the pair $\left(v_{1}, \Omega^{\prime}\right)$. By Lemma 6 , there is only one $V_{1}$ lying above $v_{1}$. Hence, by (iii) and the induction hypothesis, we have $e_{V_{1}} f_{V_{1}}=f_{V^{\prime}}$. Both facts together give $e_{V} f_{V}=n$ in view of Lemma 6 .

Lemma 9. If a subclass $\mathcal{S}$ of $D$ satisfies (iii) and if it satisfies (i) in the case of rank one, then it satisfies (i) always.

Proof. Let $(v, K)$ in $s$. We may assume $v$ of rank $>0$. To each $V$ of $K$ lying above $v$ there belongs a uniquely determined valuation ring $V^{\prime}$ of rank one, $V^{\prime} \supset V$. By Lemma 1 , we have $K_{V^{\prime}}=K_{V}$. By definition of $d_{V}$ we have therefore $d_{V^{\prime}}=d_{V}$. Because of (iii) and rank $\left(V^{\prime}\right)=1$ we have $d_{V^{\prime}}=1$, hence $d_{V}=1$. Q.E.D.

Proof of the first criterion. Let $\delta$ be the subclass of $D$ consisting of all those $(v, K)$ in $D$ such that the following condition is satisfied: If the valuation ring $V$ of $K$ lies above $v$, then the field extensions $\Omega^{i} / \mathfrak{l}^{i}$ (with the notations introduced in the introduction) are separable for $i=0, \cdots, \operatorname{rank}(V)$ -1 .

It is clear that $S$ satisfies the condition (ii) since the fields $\Omega^{i} / \mathfrak{f}^{i}$ do not change if we lift $V$ to its completion. It is also clear that $\delta$ satisfies (iii) since the set of field extensions belonging to $V^{\prime} \supset V$ is a subset of those belonging to $V$; similarly, the set of field extensions of $V_{1}$ is a subset of those belonging to $V$ by Lemma 6 .

It remains to show that $\delta$ satisfies (i). If $\operatorname{rank}(v)=1$ then $K / k=\Omega^{0} / \mathfrak{f}^{0}$ is separable by definition of $s$, hence $d_{V}=1$ by definition of the inseparability defect. Now Lemma 9 shows that $S$ satisfies (i). 
Lemma 8 shows that $S$ satisfies (iv) too. This shows that the first criterion is true, in view of the theorem.

Proof of the second criterion. Now consider the class $\mathcal{S}$ of pairs $(v, K)$ such that $k$ is finitely generated and $\operatorname{dim}(k)=\operatorname{dim}(\mathfrak{f})+\operatorname{rank}(v)$. Note that this condition is only a condition for $v$, not for $K$. Therefore we may use the phrase " $v$ belongs to $\delta$ " meaning that $(v, K)$ belongs to $\delta$ for each $K$.

Let $v$ belong to $S$ and let $r$ be the rank of $v$. Then we consider the chain $v=v^{r} \subset v^{r-1} \subset \cdots \subset v^{0}=k$ of components of $v$. By general valuation theory, we have

$$
\operatorname{dim}(\mathfrak{f})=\operatorname{dim}\left(\mathfrak{t}^{r}\right)<\operatorname{dim}\left(\mathfrak{f}^{r-1}\right)<\cdots<\operatorname{dim}\left(\mathfrak{f}^{0}\right)=\operatorname{dim}(k) .
$$

Since $\operatorname{dim}(\mathfrak{f})=\operatorname{dim}(k)-r$ it follows

$$
\operatorname{dim}\left(\mathfrak{f}^{i}\right)=\operatorname{dim}(k)-i \quad \text { for } i=0, \cdots, r .
$$

From this we deduce that (iii) is satisfied for our class $\$$.

Now we prove that (i) is satisfied too. By Lemma 9 we may assume that $r=1$. If the characteristic of $k$ is zero then $k$ is perfect, so the inseparability defects $d_{V}=1$. If the characteristic of $k$ is $>0$ then the characteristics of $k$ and $\mathfrak{t}$ are equal. Let $\mathrm{t}_{1}, \cdots, \mathrm{t}_{m}$ be a basis of transcendency for $\mathfrak{t}$ so that $\operatorname{dim}(\mathfrak{l})=m$. If we choose a representative $t_{j}$ for each $\mathrm{t}_{j}$ in $k$ then the field $R$ generated by $t_{1}, \cdots, t_{m}$ is mapped isomorphically under $\pi$. Since $\operatorname{dim}(R)$ $=m=\operatorname{dim}(\mathfrak{f})$ and $\operatorname{dim}(k)=\operatorname{dim}(\mathfrak{t})+\operatorname{rank}(v)=\operatorname{dim}(\mathfrak{f})+1$ we have $\operatorname{dim}(k / R)$ $=1$. Therefore $v$ is a valuation ring of the function field $k / R$ of one variable. Hence the inseparability defects $d_{V}=1$ by a well known result of the theory of function fields of one variable (see e.g. Artin-Whaples, Bull. Amer. Math. Soc. vol. 51 (1945) p. 484, Theorem 3).

Of course our class $\delta$ does not satisfy (ii). Therefore we consider the class $\bar{\delta}$ consisting of the $v \in \mathcal{S}$ and also of the completions $\bar{v}$ with $v \in S$. By the general theory of completions given in II it follows easily that $\overline{\mathcal{S}}$ satisfies (i)-(iii) since $\delta$ satisfies them. By Lemma $8 \bar{s}$ satisfies (iv) too. Hence, by the theorem, this proves the second criterion in the absolute case.

In the relative case, where there is a constant field $\kappa$, we have to consider the class $S_{k}$ which is defined relative to $\kappa$ exactly in the same way as $S$ is defined absolutely. The proof that $\overline{\mathcal{S}}_{x}$ satisfies (i)-(iv) is exactly the same as for $\bar{\S}$ save only that one has always to consider everything relative to $\kappa$. Q.E.D.

Added in Proof. Meanwhile, Cohen and Zariski have given another proof of the fundamental inequality (1). See: Cohen-Zariski, A fundamental inequality in the theory of extensions of valuations, Illinois Journal of Mathematics vol. 1 (1957) pp. 1-8.

Cohen and Zariski also have given a sufficient criterion for the equality sign holding in (1), namely:

(C) the integral closure of $v$ in $K$ should be a finite $v$-module. 
It does not seem to be easy to prove this criterion with the help of our Theorem 1 , since the class $\delta$ defined by the property (C) has to satisfy condition (i) which is difficult to check directly, even for rank one valuations. On the other hand, taking the result of Cohen-Zariski for granted, it follows immediately from our Lemma 5 that (i) holds for $\delta$.

UNIVERSITY OF HAMBURG, Hamburg, Germany 